\title{
Solar cycle variation of the temperature structure within the cores of coronal streamers
}

\author{
C. R. Foley ${ }^{1}$, S. Patsourakos ${ }^{1}$, J. L. Culhane ${ }^{1}$, and D. MacKay ${ }^{2}$ \\ 1 Mullard Space Science Laboratory, University College London, Holmbury St Mary, Dorking, \\ Surrey RH5 6NT, UK \\ 2 Department of Mathematical and Physical Sciences, St Andrews University, Scotland, UK
}

Received 30 July 2001 / Accepted 8 November 2001

\begin{abstract}
We use the Coronal Diagnostic Spectrometer onboard the Solar and Heliospheric Observatory (SOHO) to analyze conditions in coronal streamer structures observed close to solar minimum (1996 July 8) and near maximum (1999 August 5). We measured the intensities of emission lines from Fe IX-XV ions and found the most intense emission to be from Fe XI at solar minimum and from Fe XV at solar maximum. We then used the line ratio method with transitions in selected ions to extract the radial temperature behavior in the structures. The solar minimum peak values were about $1.4 \mathrm{MK}$ at $1.3 R_{\odot}$, while values derived close to solar maximum were consistent with the Yohkoh observations at the last maximum, displaying an apparently asymptotic temperature of around 2.2 MK above $1.2 R_{\odot}$. We discuss the observations in relation to possible mechanisms for energy deposition in large coronal structures at different phases of the solar cycle.
\end{abstract}

Key words. Sun: corona - Sun: activity - Sun: X-rays, gamma rays - stars: coronae

\section{Introduction}

Knowledge of the electron temperature in the corona and its variation with height in different regions forms an integral part of our understanding of coronal heating and solar wind acceleration. Recently the coronal temperature has been successfully measured at significant heights above the limb using the broadband filter ratio technique (Foley et al. 1996; Sturrock et al. 1996; Wheatland et al. 1997; Foley 1998; Priest et al. 1998, 2000). In these papers the radial temperature structure, $T_{\mathrm{e}}(r)$, has been used in an attempt to determine the heating function of the Sun's Corona. This work has generally found that temperature is an increasing function of height above the limb, and this result has subsequently been used to demonstrate a requirement for heating up to and including the maximum observed height range of almost 2 solar radii. Analysis of the broadband data obtained with the Yohkoh Soft $\mathrm{X}$-ray Telescope, has required an isothermal assumption which may not be realized in practice. Using this method, positive temperature gradients have been consistently reported for the quiescent regions, overlying neutral lines located in the high latitude regions.

The SOHO Coronal Diagnostic Spectrometer (CDS, Harrison et al. 1995) allows us to view the relative distribution of individual ions as function of height.

Send offprint requests to: C. R. Foley,

e-mail: carl.foley@mssl.ucl.ac.uk
Consequently, we now have the ability to examine the true distribution of plasma in many regions of the corona. In this work we evaluate the relative abundance of Fe IX through Fe XV ions for coronal streamers observed close to the solar minimum and maximum of the current solar cycle (23), on 1996 July 8 and 1999 August 5 respectively. The most abundant (adjacent in $T_{\mathrm{e}}$ ) ions were then used to establish the best representation of the apparent temperature profile with height. The derived temperature profile and its variation from solar minimum to maximum are used to infer how the corona evolves and its associated energy requirements. For comparison we have also included solar maximum data for cycle 22 (1992, October 3) obtained from Yohkoh observations using the filter ratio method (Tsuneta et al. 1991).

In the following section we describe the instrumentation. In Sect. 3, we discuss the principles behind our analysis method. Section 4 treats the observations which we obtained for solar maximum and minimum. The paper concludes with a discussion of the implications for coronal structure and heating through the solar cycle in Sect. 5, and concluding remarks in Sect. 6.

\section{Instrumentation}

The Coronal Diagnostic Spectrometer (CDS) on SOHO consists of two complementary spectrometers which make use of a common grazing incidence telescope: a Grazing 
Incidence Spectrometer (GIS); and a Normal Incidence Spectrometer (NIS). Together, GIS and NIS cover most of the wavelength range from $150 \AA$ to $785 \AA$ (Harrison et al. 1995). The GIS system has four detectors, with spectral ranges 151-221 $\AA, 256-341 \AA, 393-492 \AA$ and $656-$ $785 \AA$, while the NIS covers two ranges: $308-381 \AA$ and 513-633 Å.

The NIS uses for its detector an intensified CCD camera. This makes use of a micro-channel plate with a phosphor converter which intensifies and converts the incident EUV to visible light. This is then focussed by a lens onto a CCD.

The GIS detectors (Breeveld et al. 1992) are openfaced microchannel plate (MCP) detectors in a Z stack configuration with the output charge of the MCP directed on to a charge division read-out. The detectors operate in photon counting mode, and this allows events to be discriminated by pulse height, thereby allowing cosmic ray hits to be excluded from the accumulated spectra. The GIS is therefore able to obtain deep exposures relatively free of cosmic ray background.

The Yohkoh Soft X-ray Telescope (SXT, Tsuneta et al. 1991) provides full-Sun images in the range 4-60 A. Use of pairs of filters allows the estimation of electron temperature assuming that the plasma sampled is isothermal.

Coronagraphic images of the streamers that were observed were obtained with the SOHO Large Angle Solar Coronagraph (LASCO, Brueckner et al. 1995) and with the ground-based instrument on Mauna Loa (Fisher et al. 1981).

\section{Analysis method}

\subsection{Spectroscopic diagnostics}

In this work we use the intensities of individual emission lines in the EUV regime to determine the properties of the Sun's corona to over one solar radius above the solar limb. Assuming optically thin radiation the intensity of an emission line which we observe can be represented by (Mason \& Fossi 1994)

$I=\int_{l} A_{\mathrm{el}} C\left(T, n_{\mathrm{e}}\right) n_{\mathrm{e}}^{2} \mathrm{~d} l$,

where the integral is over the line of sight (1); $A_{\mathrm{el}}$ is the absolute abundance of the element and $C\left(T, n_{\mathrm{e}}\right)$ is the contribution function of the emitting line at electron density $n_{\mathrm{e}}$. We used the CHIANTI spectral package (Dere et al. 1997) for calculating the contribution functions $C\left(T, n_{\mathrm{e}}\right)$, adopting Grevesse \& Sauval (1998) coronal abundance values and Mazzotta et al. (1998) ionization equilibria.

As mentioned in the introduction, observations of the temperature profile in the corona have been obtained with the Yohkoh SXT near the maximum of cycle 22 (1992, October 3). These observations use the ratio of the signal recorded in two broadband filter images to determine the weighted isothermal temperature of the observed region. By taking the calculated intensity ratios using emission

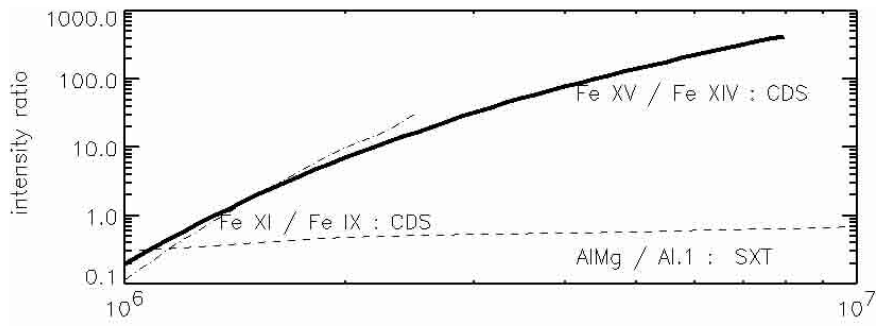

(a)

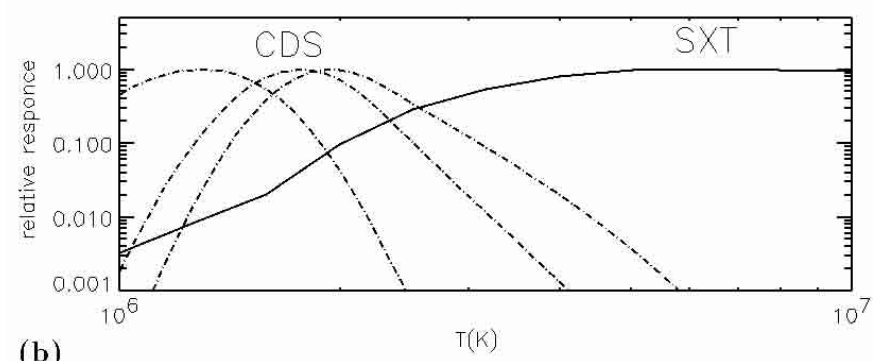

Fig. 1. a) Line ratios and Yohkoh SXT filter ratio used for the diagnostics in this paper. b) The contribution functions $C\left(T, n_{\mathrm{e}}\right)$ for Fe XI, XIV, XV lines and the Yohkoh SXT thin aluminum filter. These have each been normalized to their peak value.

lines from the same element (e.g. Fe, see Fig. 1), we can estimate $T_{\mathrm{e}}$ values which are free from uncertainty in $A_{\mathrm{el}}$ but do rely on the accuracy of the ionization equilibrium calculation. The strength of using this spectroscopic technique as compared to the filter ratio method is highlighted in Fig. 1. In this figure we plot as a function of temperature (a) several line and filter intensity ratios for CDS and SXT respectively and (b) the normalized (to their peak value) temperature responses for several CDS spectral lines and an SXT filter. The line ratios are $\sim 1000$ times more sensitive to temperature change than are the broadband filter ratio observations of the Yohkoh SXT.

An additional benefit of using emission line diagnostics in this region is that the contribution functions are more constrained in temperature. This allows the average emission measure for a range of temperatures to be estimated. Although this is an average emission measure, it does indicate the approximate amount of material at each temperature which would produce the observed emission line intensities (e.g. see Griffiths \& Jordan 1998).

\subsection{Validity of the ionization equilibrium assumption}

Our derivation of electron temperatures will be made with the assumption of ionization-equilibrium. However, since mass flows take place in streamers, these may induce some departures from the ionization-equilibrium due to slow solar wind flow. Therefore the transit times $\tau_{\text {transit }}$ associated with mass flows should be considered as they may induce some departures from the ionizationequilibrium (e.g., Dupree et al. 1979). The ionization time $\tau_{\text {ioniz }}$ is a function of both the electron temperature and density. We have used here the analytic fits given in 

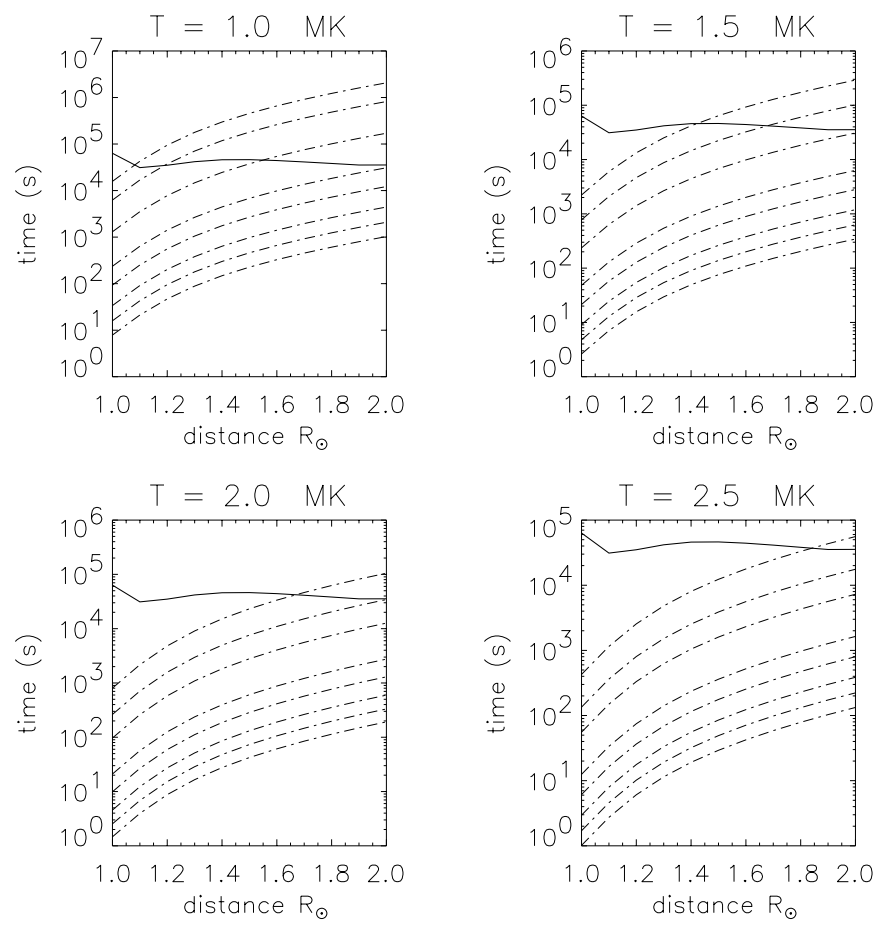

Fig. 2. Transit (in thick lines) and ionization times (in dashesdots) as a function of the solar distance for Fe IX to Fe XVI. The ionization times were calculated by assuming a constant temperature which is indicated in each panel. Ionization times for Fe IX to Fe XVI run from the bottom to the top in the figure.

Shull \& Van Steenberg (1982). The transit times $\tau_{\text {transit }}$ associated with mass flows may be defined as:

$\tau_{\text {transit }}=\frac{n_{\mathrm{e}}}{v \mid \frac{\mathrm{d} n_{\mathrm{e}} \mid}{\mathrm{d} r}}$

where $v$ is the flow speed of the solar wind and $n_{\mathrm{e}}$ the electron density. $\tau_{\text {transit }}$ is essentially the time taken for the flowing plasma to traverse a density scale-height.

The slow solar wind radial velocity $v$ was determined by assuming mass flux conservation between the inner corona and 1 AU. We used the standard form of the cross-sectional area of a flux tube given by Kopp \& Holtzer (1976) and adapted it for slow speed wind emanating from streamers by using the parameters given in Withbroe (1988). We used the value of $n_{\mathrm{p}} v=3.8 \times$ $10^{8} \mathrm{~cm}^{-2} \mathrm{~s}^{-1}$ measured in-situ by Ulysses in the ecliptic plane (Goldstein et al. 1996). For the $n_{\mathrm{e}}$ radial profile, we used the corresponding relation for streamers given by Guhathakurta et al. (1996). We finally assumed a fully-ionized plasma with $10 \%$ helium which results in $n_{\mathrm{p}}=1.2 n_{\mathrm{e}}$. We found a slow solar wind velocity that does not exceed $20 \mathrm{kms}^{-1}$ at $2 R_{\odot}$.

The results for the calculated $\tau_{\text {ioniz }}$ and $\tau_{\text {transit }}$ for the distance range $1-2 R_{\odot}$ and for four different values of the temperature, which was assumed to be constant in the above region, are shown in Fig. 2. Ionization times tend to increase with distance due to the decrease of electron density, while transit times decrease with distance as

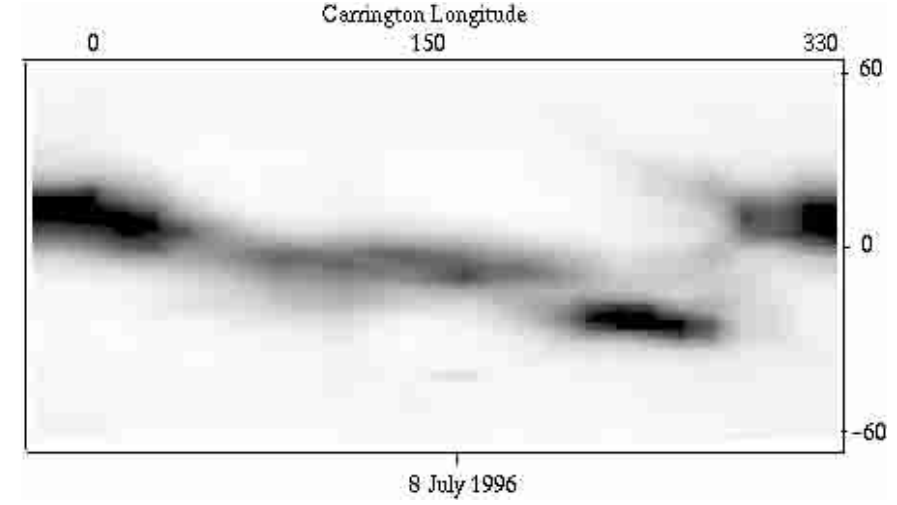

Fig. 3. Lasco $\mathrm{C} 2$ synoptic map generated by extracting and averaging the daily signal at a height of $2.2 R_{\odot}$, between $\pm 60^{\circ}$.

a consequence of the solar wind acceleration. Ionization times are smaller than transit times for the lower ionization stages of iron (Fe IX-Fe XIII) and for all the distances considered. However we find, for Fe XIV, Fe XV and Fe XVI at $T<2 \mathrm{MK}$, that $\tau_{\text {ioniz }}>\tau_{\text {transit }}$ for most of the distances covered by our observations. Therefore under the above conditions non-ionization equilibrium effects could be relevant for the above Fe ions. On the other hand, for temperatures greater that $2 \mathrm{MK}$, ionization times begin to exceed the transit times beyond a distance of about $1.7-1.8 R_{\odot}$, which is the largest radial distance of our solar maximum observations. Therefore, since the $I(\mathrm{Fe} \mathrm{XV}) / I(\mathrm{Fe} \mathrm{XIV})$ and $I(\mathrm{Fe} \mathrm{XVI}) / I(\mathrm{Fe} \mathrm{XIV})$ ratios lead to temperatures larger than $1.9 \mathrm{MK}$, (see Sect. 4.3), we can assert that ionization equilibrium exists for these ions under solar maximum conditions. We have also calculated the recombination times and find that even at $2 R_{\odot}$ where the lowest density plasma to be encountered in this work is found, the recombination time is at least a factor ten below the transit time for the entire temperature range being considered.

\section{Observations}

\subsection{Solar minimum: 1996 July 8}

During the middle part of 1996 the solar cycle reached its minimum phase of activity. The corona was dominated by two large polar coronal holes, with a relatively simple large-scale coronal streamer extending between them. We constructed a synoptic map using LASCO C2 data at a height of $2.2 R_{\odot}$ which we present in Fig. 3 . This is centered on the simple streamer, observations of which we obtained using the Grazing Incidence Spectrometer (GIS) on SOHO on 1999 July 8. The synoptic map demonstrates that the quiescent streamer was symmetric about the limb in longitude at the time of our observations, and that there are no other structures contributing to or biasing our measurements.

In Fig. 4 we have illustrated the region as it appeared in one of these images. We have filled the occulted region with an image obtained from the Mauna Loa MK3 


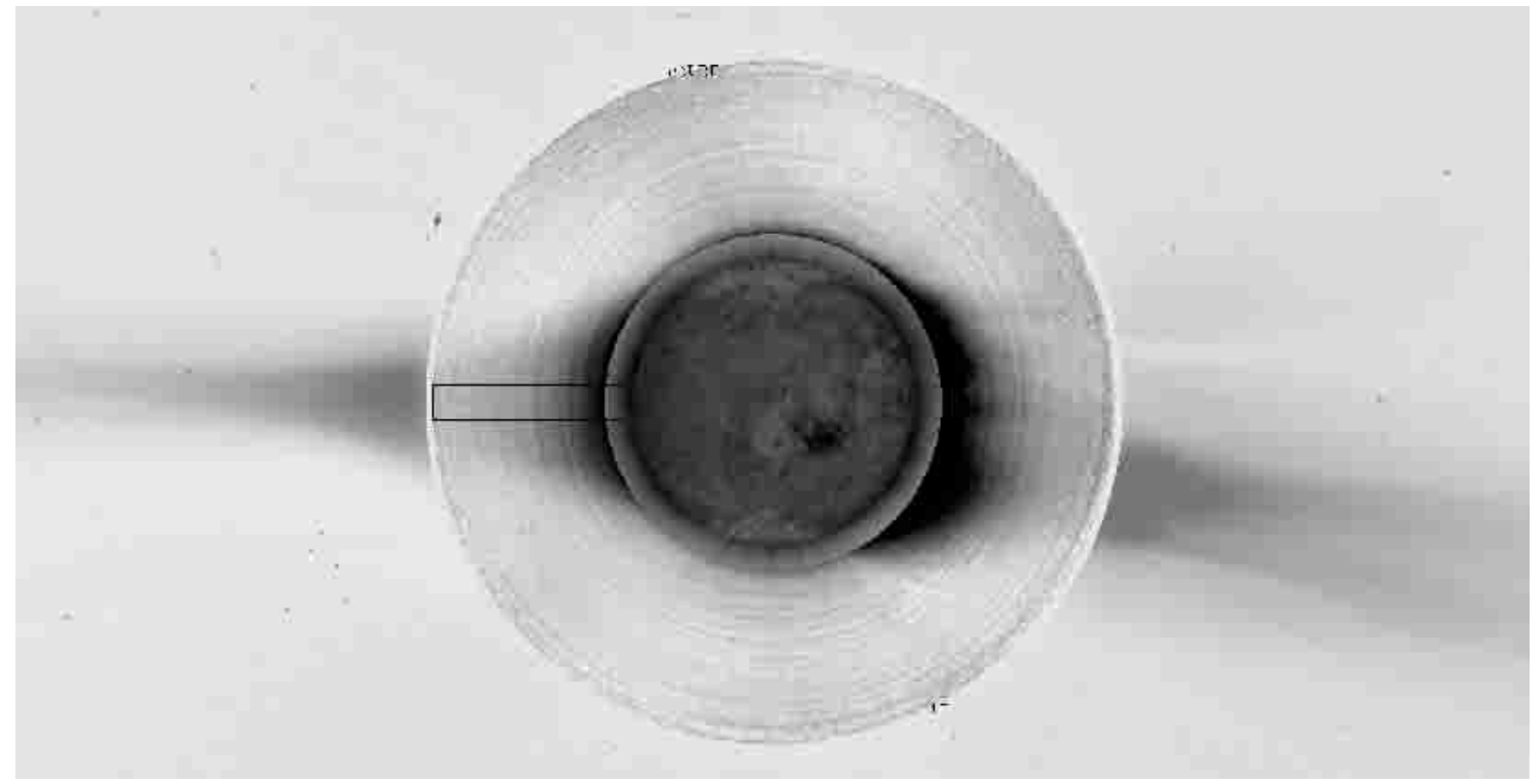

Fig. 4. Composite image of the Sun that was obtained on 1996 July 8. The central part is an EIT $284 \AA$ image, outside that is a Mauna Loa coronagraph image, and the outermost region a LASCO C2 image.

Table 1. Study summary for solar minimum observations.

\begin{tabular}{|c|c|c|c|c|}
\hline \multirow[t]{2}{*}{ Coordinates } & \multirow{2}{*}{$\begin{array}{l}\text { Height } \\
\left(R_{\odot}\right) \\
\end{array}$} & \multirow{2}{*}{$\begin{array}{c}\text { Exposure } \\
(\mathrm{s})\end{array}$} & \multicolumn{2}{|c|}{$\begin{array}{c}\text { Raster } \\
\text { Positions (spacing) }\end{array}$} \\
\hline & & & $($ solar $-x)$ & (solar- $y)$ \\
\hline$-1120,0$ & 1.17 & 20 & $30\left(8^{\prime \prime}\right)$ & $3\left(100^{\prime \prime}\right)$ \\
\hline$-1360,0$ & 1.42 & 40 & $30\left(8^{\prime \prime}\right)$ & $3\left(100^{\prime \prime}\right)$ \\
\hline$-1600,0$ & 1.67 & 80 & $30\left(8^{\prime \prime}\right)$ & $3\left(100^{\prime \prime}\right)$ \\
\hline$-1840,0$ & 1.92 & 160 & $30\left(8^{\prime \prime}\right)$ & $3\left(100^{\prime \prime}\right)$ \\
\hline$-2080,0$ & 2.17 & 160 & $30\left(8^{\prime \prime}\right)$ & $3\left(100^{\prime \prime}\right)$ \\
\hline
\end{tabular}

Coronagraph, while the disc region is used to display an EIT $284 \AA$ image. The area which our observations cover is delineated by the rectangle and was stable for a few Carrington rotations. For some of these it has already been studied by others (Raymond et al. 1997; Gibson et al. 1999; Li et al. 1998).

The observational sequence which we used to study the region is summarized in Table 1 . This was performed using the $8^{\prime \prime} \times 50^{\prime \prime}$ slit and produced five rasters of size $240^{\prime \prime} \times 250^{\prime \prime}$. We used the standard procedures (gis_smooth, ghost_buster, and gis_calib) which are distributed as part of the CDS software, to correct and intensity calibrate the data. These routines use the results of the laboratory calibration of the CDS which is described by Lang et al. (2000). This calibration confirms the flux of any detected emission line to an accuracy of $30 \%$ which applies to all the observations we describe from the CDS instrument here. These calibrations have been confirmed in-flight by Landi et al. (1999).
We have summed data from each of the five rasters to produce an average count rate for each radial position. We fitted selected regions using the $c$ fit routine which is distributed as part of the Solarsoft package. This allows a structure to be defined which contains the lines and the allowed range of parameters of the fit, such as width, position and intensity. The results of these fits for each of the raster positions are presented in Table 2.

In order to understand the distribution of plasma we have plotted the loci of the emission measure (see e.g. Griffiths \& Jordan 1998) for each ion from Table 2 as a function of temperature in Fig. 5. These loci represent from left to right, the emission measure which would reproduce our observed flux for Fe IX-Fe XV within the height range $1.05-1.3 R_{\odot}$. If the plasma was isothermal, all the curves should intersect at the correct single value of emission measure. It is clear from the figure that this is not the case which suggests that the plasma located in the height range is distributed in temperature between 1-1.5 MK.

To investigate the variation of temperature as a function of height we have used the line intensity ratio which is most sensitive to temperature changes for the bulk of the plasma in this temperature regime namely $I(\mathrm{Fe} \mathrm{IX}) / I(\mathrm{Fe} \mathrm{XI})$. We present the results of this analysis in Sect. 4.3 along with similar calculations for the solar maximum.

\subsection{Solar maximum: 1999 August 5}

Solar maximum of the current solar cycle was characterized by the appearance of bright coronal streamers located 
Table 2. Averaged photon fluxes obtained for our solar minimum observations. The laboratory wavelengths are those from Dere et al. (1997).

\begin{tabular}{|l|c|r|r|r|r|r|}
\hline $\begin{array}{c}\text { Emission } \\
\text { Line }\end{array}$ & $\begin{array}{c}\text { Laboratory } \\
\text { wavelength }\end{array}$ & \multicolumn{5}{|c|}{$\begin{array}{c}\text { Intensity } \\
\text { photons } \mathrm{cm}^{-2} \mathrm{~s}^{-1} \mathrm{str}^{-1}\end{array}$} \\
\hline & $(\AA)$ & $1.05-1.3 R_{\odot}$ & $1.3-1.55 R_{\odot}$ & $1.55-1.8 R_{\odot}$ & $1.8-2.05 R_{\odot}$ & $2.05-2.3 R_{\odot}$ \\
\hline Fe IX & 171.07 & 2107 & 38.7 & 15.5 & 9.34 & 6.77 \\
Fe X & 184.54 & 624 & 8.97 & 2.90 & 1.35 & 0.91 \\
Fe X/XII & 190.04 & 296 & 5.08 & 1.59 & 0.80 & 0.57 \\
Fe XI & 188.22 & 2289 & 58.8 & 16.6 & 7.78 & 5.25 \\
Fe XII & 186.88 & 264 & 5.39 & 1.91 & 1.08 & 0.71 \\
Fe XIII & 202.04 & 1125 & 65.7 & 16.3 & 8.00 & 3.94 \\
Fe XIII & 203.79 & 182 & 4.34 & 0.87 & 0.65 & 0.28 \\
Fe XIV & 334.17 & 140 & 16.8 & 3.84 & 2.25 & 1.19 \\
Fe XV & 284.16 & 470 & 61.0 & 14.9 & 8.75 & 3.46 \\
\hline
\end{tabular}

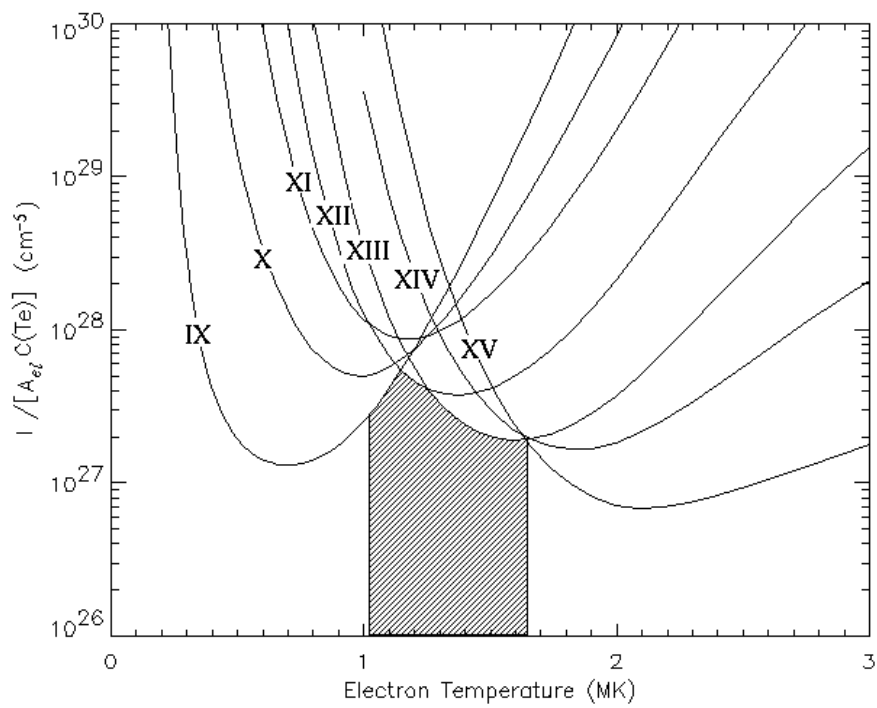

Fig. 5. Emission measure loci of each Fe ion from Table 2 for $r=1.05-1.3 R_{\odot}$. The hatched region represents the probable distribution of plasma in the observed region.

close to the poles. These structures appear to have evolved from the decaying magnetic field from the first high latitude spots of the cycle. During 1999 August 5, we performed observations of such a compact streamer. In Fig. 6 we show a composite view using an enhanced SXT image and a MK4 coronagraph image which was obtained from the Mauna Loa solar observatory. The streamer was observed to have evolved directly from active region AR 8657, and remained connected via an extended arcade which can be seen to increase in altitude away from the active region center.

To study this region we constructed an observing sequence which rastered over the whole streamer and included data from both the NIS and GIS. The observational sequence used for each detector is summarized in Table 3.

The NIS as mentioned earlier, covers a complementary wavelength range and has the ability to image a region. We coarsely sampled the region (as indicated in the bottom section of Table 3$)$ with slit $5\left(4^{\prime \prime} \times 240^{\prime \prime}\right)$ along which we

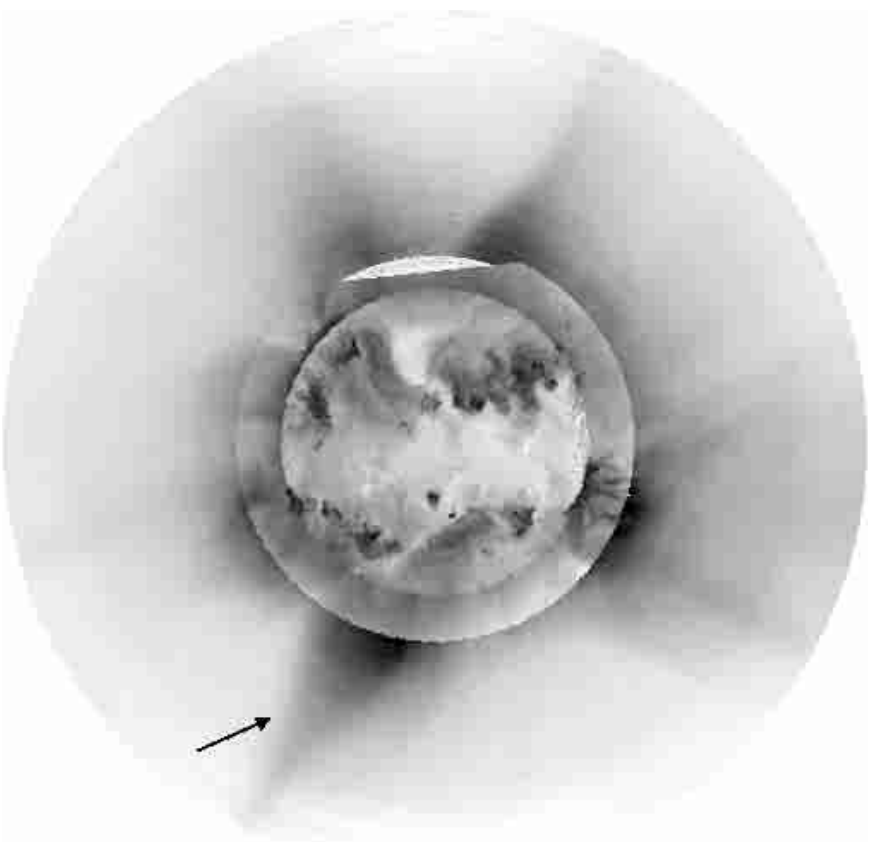

Fig. 6. Composite view of the corona formed from an enhanced composite Yohkoh SXT image (central region) and a Mauna Loa MK4 coronagraph image (outer region) on 1999 August 5. The streamer which we observed is indicated by the arrow was centered at a Carrington longitude of $80^{\circ}$ of rotation 1952 .

summed every 8 pixels, to produce a macropixel. We did this to reduce the required telemetry transmission bandwidth and thus allow the complete spectra of NIS 1 and NIS 2 to be returned within a reasonable time and without additional telemetry overheads. The NIS observations allow us to check the calibration of our lines and to observe the emission variation all the way to the limb, where the intensity would normally exceed threshold limits of the GIS detectors.

We corrected the data using the same procedure which we described in the preceding section. There is no evidence for significant aging of the GIS detectors over the course of mission, and we have therefore used the preflight flat field for the GIS. This is not the case for the NIS which requires 
Table 3. Study summary for solar maximum observations obtained on 1999 August 5. The upper part of the table refers to the GIS observations whilst those below the dividing line represent the NIS observations. The NIS was used to cover the lower corona and Limb.

\begin{tabular}{|r|c|c|c|c|}
\hline Coordinates & Height & Exposure & \multicolumn{2}{|c|}{ Raster } \\
& & & positions $($ spacing $)$ \\
\hline & $\left(R_{\odot}\right)$ & $(\mathrm{s})$ & solar- $x$ & solar-y \\
\hline$-920,-1170$ & 1.55 & 50 & $30\left(32^{\prime \prime}\right)$ & $4\left(61^{\prime \prime}\right)$ \\
$-920,-1020$ & 1.43 & 20 & $30\left(8^{\prime \prime}\right)$ & $5\left(51^{\prime \prime}\right)$ \\
$-920,-780$ & 1.26 & 10 & $30\left(8^{\prime \prime}\right)$ & $5\left(51^{\prime \prime}\right)$ \\
$-680,-1383$ & 1.60 & 50 & $8\left(32^{\prime \prime}\right)$ & $4\left(61^{\prime \prime}\right)$ \\
$-680,-1215$ & 1.45 & 20 & $30\left(8^{\prime \prime}\right)$ & $5\left(51^{\prime \prime}\right)$ \\
$-680,-975$ & 1.24 & 10 & $30\left(8^{\prime \prime}\right)$ & $5\left(51^{\prime \prime}\right)$ \\
$-440,-1535$ & 1.66 & 50 & $8\left(32^{\prime \prime}\right)$ & $4\left(61^{\prime \prime}\right)$ \\
$-440,-1335$ & 1.46 & 20 & $30\left(8^{\prime \prime}\right)$ & $5\left(51^{\prime \prime}\right)$ \\
$-440,-1095$ & 1.23 & 10 & $30\left(8^{\prime \prime}\right)$ & $5\left(51^{\prime \prime}\right)$ \\
$-200,1375$ & 1.45 & 20 & $30\left(8^{\prime \prime}\right)$ & $5\left(51^{\prime \prime}\right)$ \\
$-200,-1135$ & 1.20 & 10 & $30\left(8^{\prime \prime}\right)$ & $5\left(51^{\prime \prime}\right)$ \\
\hline$-680,-975$ & 1.24 & 50 & $30\left(8^{\prime \prime}\right)$ & $18\left(13^{\prime \prime}\right)$ \\
$-680,-735$ & 1.04 & 50 & $30\left(8^{\prime \prime}\right)$ & $18\left(13^{\prime \prime}\right)$ \\
$-440,-1095$ & 1.23 & 50 & $30\left(8^{\prime \prime}\right)$ & $18\left(13^{\prime \prime}\right)$ \\
$-440,-855$ & 1.00 & 50 & $30\left(8^{\prime \prime}\right)$ & $18\left(13^{\prime \prime}\right)$ \\
\hline
\end{tabular}

Table 4. Photon fluxes obtained for the solar maximum observations with the GIS. These are the averaged counts for the region indicated and at a latitude of $-60^{\circ}$ to $-70^{\circ}$ for the streamer illustrated in Fig. 6.

\begin{tabular}{|c|c|c|c|}
\hline $\begin{array}{l}\text { Emission } \\
\text { Line }\end{array}$ & $\begin{array}{l}\text { Laboratory } \\
\text { wavelength }\end{array}$ & \multicolumn{2}{|c|}{$\begin{array}{c}\text { Intensity } \\
\times 10^{8} \text { photons } \mathrm{cm}^{-2} \mathrm{~s}^{-1} \mathrm{str}^{-1}\end{array}$} \\
\hline & $(\AA)$ & $1.05-1.3 R_{\odot}$ & $1.3-1.55 R_{\odot}$ \\
\hline Fe IX & 171.07 & 674 & 215 \\
\hline $\mathrm{Fe} \mathrm{XI}$ & 188.22 & 919 & 188 \\
\hline Fe XII & 186.88 & 166 & 34.3 \\
\hline Fe XIII & 202.04 & 3706 & 702 \\
\hline Fe XIII & 203.79 & 385 & 52.0 \\
\hline Fe XIV & 211.03 & 3541 & 694 \\
\hline Fe XV & 417.75 & 2500 & 530 \\
\hline
\end{tabular}

a flat field to correct for the Long Term Gain Depression (LTGD). We thus use the NIS to check the intensities of the lines in the overlap region $\left(1.05-1.20 R_{\odot}\right)$, and to confirm the absolute intensity calibration of similar lines found in both the NIS (Fe XIV $334.17 \AA$, Fe XV $327.06 \AA$ ) and GIS (Fe XIV 211.32 A, Fe XV 417.75) detectors.

The brightest lines we observed and for which we could obtain fits, are summarized in Table 4. These included lines which appeared in GIS detectors 1 and 3, Fe XIV and Fe XV located at $211.3 \AA$ and $417.75 \AA$ respectively. In Fig. 7 we plot loci of the emission measure for each ion from Table 2 as a function of temperature as we did for the solar minimum observation. The difference in the streamer temperature is striking in the plot. The emission is dominated by plasma in the region of $2 \mathrm{MK}$; in

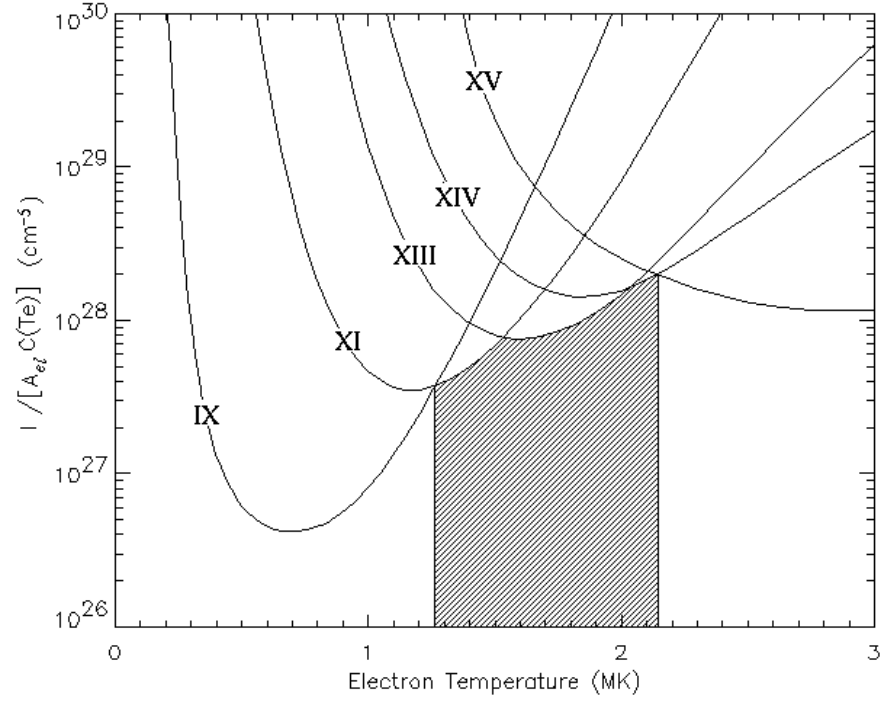

Fig. 7. Emission measure loci for solar maximum data of each Fe ion from Table 4 for $r=1.05-1.3 R_{\odot}$ and latitude $=-60^{\circ}$ to $-70^{\circ}$. The hatched region represents the probable plasma distribution within the observed region. This distribution is both hotter and denser than that presented in Fig. 7 for solar minimum. The Fe XIII, XIV, and XV all intersect close to $2.1 \mathrm{MK}$

fact this component is enhanced by an order of magnitude compared to the solar minimum values, while the cooler component (Fe IX, XI $\sim 1 \mathrm{MK}$ ) in this region is effectively halved.

The origin of the different plasma components is illustrated in Fig. 8. In this figure the streamer is bright and easily resolved in the hotter emission lines Fe XIV-XVI, and faintly in Fe XIII. Fe XI is enhanced at the boundary to the streamer which we have indicated by the arrow in the figure. There appears to be no Fe XI plasma within the streamer which demonstrates that the streamer is hotter than 1.3 MK, the peak temperature of formation for this ion.

To investigate the variation of temperature as a function of height through the core $\left(-60^{\circ}\right.$ to $-70^{\circ}$ latitude $)$ of this solar maximum streamer we have used the line intensity ratio which is most sensitive to temperature changes for the bulk of the plasma in this temperature regime namely $I(\mathrm{Fe} \mathrm{XV}) / I(\mathrm{Fe} \mathrm{XIV})$ and $I(\mathrm{Fe} \mathrm{XVI}) / I(\mathrm{Fe} \mathrm{XIV})$ for the GIS and NIS respectively. We present the results of this analysis in the following section along with similar calculations for the solar minimum.

\subsection{Line ratio temperatures}

In Fig. 9 we present the temperature estimates that result from the CDS line fits and line ratios. We also plot the radial temperature structure from the SXT filter ratios which were presented in Foley (1998). We note the excellent agreement between values obtained using the broadband filter ratio technique for the solar maximum (cycle 22) and the observations we have presented here from 


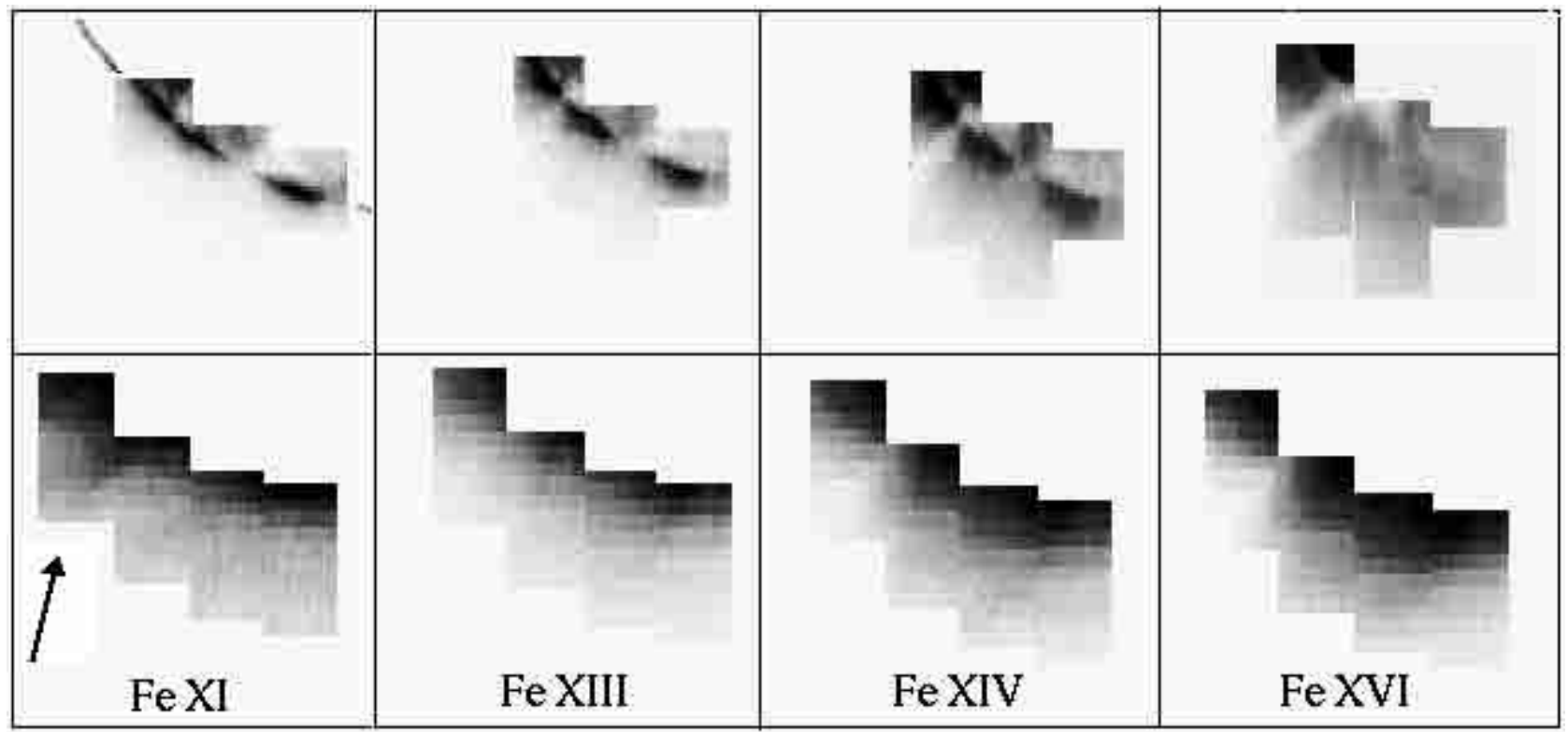

Fig. 8. Monochromatic images constructed of the observed region. The upper panel corresponds to the NIS observations, and the lower panel corresponds to the GIS observations as summarized in Table 3. In the top left hand panel we have indicated the location of the solar limb. It is interesting to note that the Fe XI emission appears to dominate at the edge of the streamer where the field is expected to be open (arrowed).

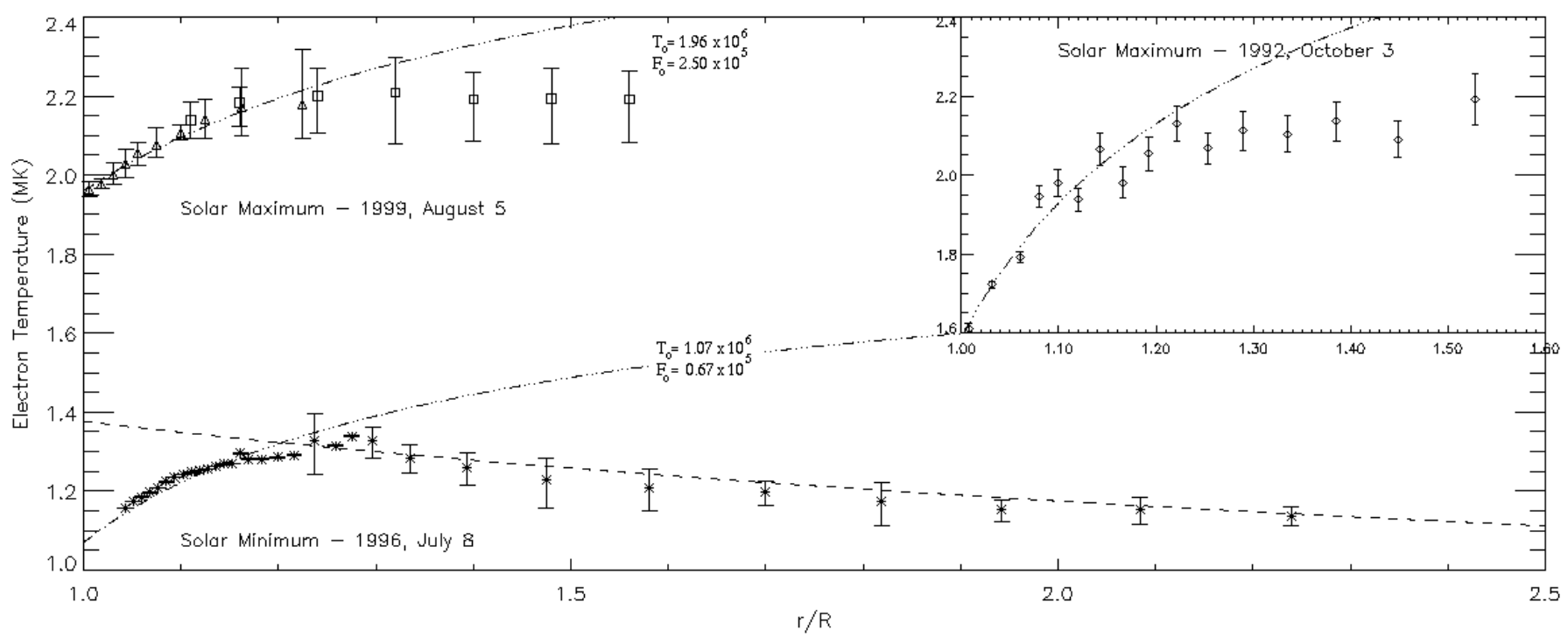

Fig. 9. The radial temperature structure determined for solar maximum (1999 August 5, triangles - NIS; squares - GIS) and solar minimum (1996 July 8, asterisks). The values from Foley (1998) are over plotted as diamonds in the inset panel and were determined through a similar region at the last solar maximum. The similarity between the two maximums is surprising given the degree of systematic uncertainty associated with each plot. The error bars of each point represent the statistical error of the measurements. The standard error is associated with the uncertainty in the relative calibration of each emission line/passband. In the case of CDS the absolute calibration this is approximately $30 \%$ for the GIS and equates to approximately 1 MK. We have overplotted a conserved heat flux model from Sturrock et al. (1996), with the fitted values indicated. For solar minimum this includes an outward heat flux from Owocki et al. (1983).

the current maximum (cycle 23) using CDS. In evaluating the line ratio $I(\mathrm{Fe} \mathrm{XV}) / I(\mathrm{Fe} \mathrm{XIV})$ from the GIS we found the temperatures in fact were in the region of $2.5 \mathrm{MK}$. We attributed this to the calibration of the detectors and adopted the NIS calibration by normalizing our ratio to that observed with the NIS.

\section{Discussion}

\subsection{Relevance to other observation}

The radial temperature structure we derive here agrees well with typical values which were found during the last 
solar maximum which are summarized in Foley (1998). This suggests that the assumptions which were used in deriving the temperatures from the Yohkoh SXT filter ratio data were valid during the solar maximum. These assumptions were associated with the use of two broadband filters which limit the analysis to a weighted isothermal approximation for each position.

Our other finding of an increase in the coronal temperature from solar minimum to maximum, is broadly consistent with electron density distributions obtained from eclipse observations during solar minimum and maximum periods (e.g., Gabryl et al. 1999). These authors found that when moving from solar minimum to maximum the slope of the electron density radial distribution in streamers flattens. This would imply, by assuming hydrostatic equilibrium, that the electron temperature (assumed equal to the ion temperature) should increase from solar minimum to solar maximum. This is exactly what we observe.

Gibson et al. (1999) reports the densities and temperatures of the same coronal streamer observed 1.5 Carrington rotations later on 1996 August 17. This was determined from the density gradients found from the LASCO C2 and Mauna Loa MK 3 Coronameter data. They found temperatures which peaked at 1.5 MK and a location $1.3 R_{\odot}$. These results are consistent with those we have found here spectroscopically. This suggests that the cores of solar minimum quiescent streamers are in hydrostatic equilibrium.

Departure from hydrostatic equilibrium is something which is often cited as a reason why soft X-ray emission gradients cannot be fitted for coronal streamer data. However, it is noted that solar minimum streamers may be considered to be in hydrostatic equilibrium from the work here. It is interesting to note that in Sturrock et al. (1996) that the analysis of a structure which appears to be a leg of a large scale loop was consistent with a hydrostatic barometric atmosphere.

\subsection{Streamer structure and the location of the temperature maximum}

In Fig. 10 we present a potential field extrapolation of the magnetic field in the region of interest as would be seen when viewed on the limb. To obtain the extrapolation, the synoptic magnetogram CR 1911 was taken from the $\mathrm{NSO} /$ Kitt Peak database and a potential field $(\nabla \times \boldsymbol{B}=0)$ deduced. A source surface is placed at $2.5 R_{\odot}$ where the field is assumed to become radial. This in a crude manner simulates the effect of the solar wind opening up the field which then extends into interplanetary space. From the image it can be seen that near the equator at low heights there are many loops that lie along the line of sight. With increasing height, until about $r \sim 1.4 R_{\odot}$, the loops become more concentrated, longer and extend from the higher latitudes of each hemisphere. We can see from Fig. 9 that our temperature maximum is located around

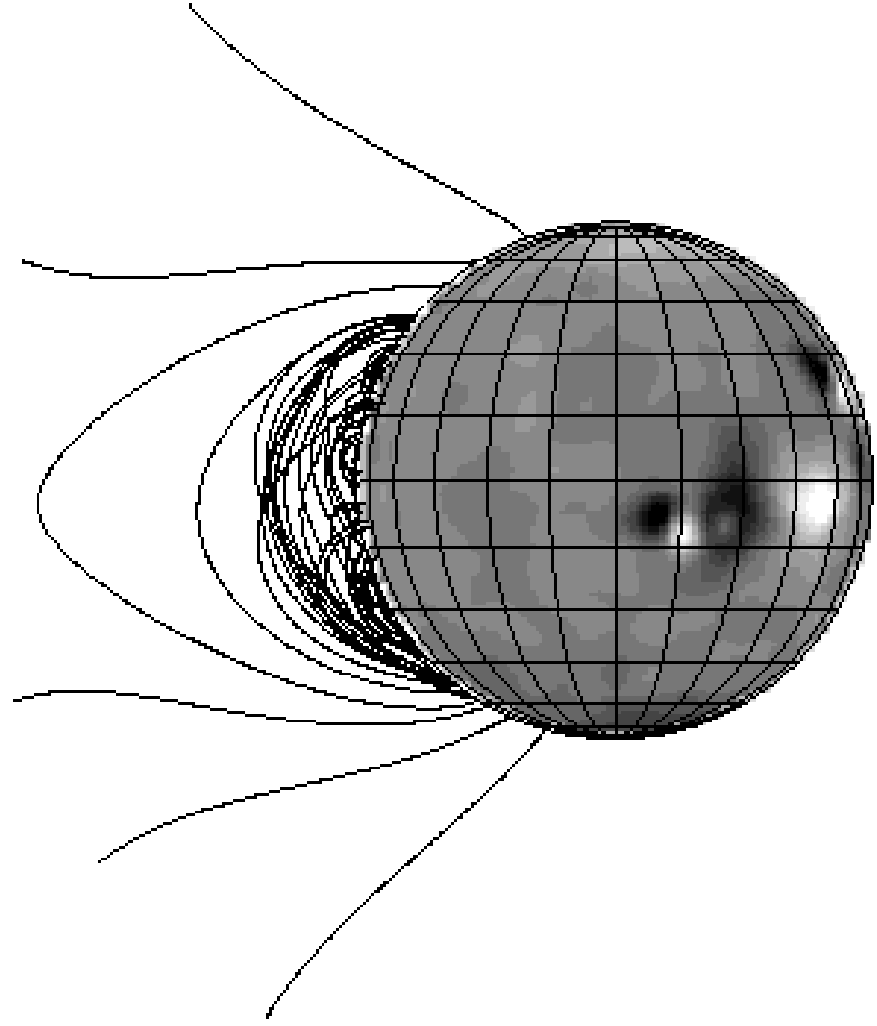

Fig. 10. Potential field extrapolation of the magnetic field in the region of interest on 1996, July 8.

the tops of these larger loops, and declines at greater heights.

We have over-plotted in Fig. 9 (dashed curve) the temperature profile anticipated if the decline from this height was that expected for outward conserved conductive loss (see Owocki et al. 1983)

$T(r)=T_{\circ}\left(r / R_{\odot}\right)^{-\frac{2}{7}}$

where $T_{\circ}$ is the electron temperature at the base of the corona. We also have over-plotted in Fig. 9 (dashed-dots curve) the temperature which corresponds to a conserved inward heat flux (such as that proposed by Sturrock et al. 1996) namely,

$T(r)=\left(T_{\circ}^{\frac{7}{2}}+7 \frac{R_{\odot} F_{\circ}}{2 a r}\left(r-R_{\odot}\right)\right)^{\frac{2}{7}}$

where $F_{\circ}$ is the heat flux at the base of the corona and $a$ is the coefficient of thermal conductivity (Spitzer 1962).

Measurements of the plasma beta value in the cores of streamers have been performed by Li et al. (1998). They found that the plasma pressure was the dominant force in streamer cores. In light of this and our outwardly declining temperature gradient, it would seem probable that we are viewing the initiation of the slow speed wind from the tops of loops. The fact that we observe a temperature increase within the cores of coronal streamers would imply that the heating source for the streamer must lie at or above the location of the temperature maximum e.g. at the tops 
of the large transequatorial loops that comprise the large scale structure of the streamers.

Raymond et al. (1997), Raymond et al. (1998) and Feldman et al. (1999) report a depletion of high FIP elements at solar minimum within quiescent streamers. They attribute this to a gravitational settling. This is consistent with the view drawn in the previous paragraph, where the principal energy source is from the heating of the loops embedded within streamers. The material which fills coronal streamers may also originate within these loops. This may account for the often reported departure from normal hydrostatic equilibrium for coronal streamer structures (e.g. see Aschwanden \& Nitta 2000). Although this may also be directly related to the geometry.

\subsection{Coronal heating}

The role of coronal magnetic field in coronal heating is well established with many different heating mechanisms being distinguished by their scaling with the field strength (Priest et al. 1998, 2000). In such models the coronal heating rate is proportional to $B^{\alpha}$, with $\alpha$ being a function of the specific mechanism of magnetic energy build-up, release and dissipation. These mechanisms were recently reviewed by Mandrini et al. (2000), who compiled the values of $\alpha$ expected for different magnetic-based heating scenarios. Our aim here is to calculate an approximate value for $\alpha$ so as to gain insights on which magnetic heating models may be more relevant. By assuming that the heating sets up a conductive flux in the corona and by noting from Fig. 9 that the temperature scale heights are more or less identical for solar minimum and maximum, we can write, to first order, that

$$
\left(\frac{T_{\max }}{T_{\min }}\right)^{\frac{5}{2}}=\left(\frac{B_{\max }}{B_{\min }}\right)^{\alpha}
$$

where $T_{\max }, B_{\max }$ and $T_{\min }, B_{\min }$ are the maximum coronal temperature and the magnetic field strength for solar maximum and minimum respectively. By assuming $\frac{B_{\max }}{B_{\min }} \approx 2$ (e.g. Pevtsov \& Acton 2001) and $\frac{T_{\max }}{T_{\min }} \approx 1.5$ from Fig. 9, we find that $\alpha \approx 1.5$. This value of $\alpha$ compares well with the $\alpha$ predicted by "direct current" stressing models based on reconnection and turbulence with constant dissipation coefficients (cf. models 5 and 11 in the Mandrini et al. 2000 compilation).

\subsection{Transition region emission}

In a recent paper Schühle et al. (2000), found by using SUMER data, an increase in the transition region (TR) emission of up to a factor of 2 from solar minimum to solar maximum. This behavior could be related to the observed increase of the coronal temperature demonstrated in the previous sections. Transition region models for the quiet Sun (e.g., Gabriel 1976) indicate that TR radiative losses are powered from the coronal conductive flux. By noting from Fig. 9 that the temperature scale heights are more or less identical in solar minimum and maximum, we can make the approximation that the TR radiative losses $L_{\text {rad }}$ would scale as $T_{\max }^{\frac{5}{2}}$ where $T_{\max }$ is the maximum coronal temperature. Therefore, we have (Withbroe 1988)

$\frac{L_{\max }}{L_{\min }}=\left(\frac{T_{\max }}{T_{\min }}\right)^{\frac{5}{2}}$

where $L_{\max }, T_{\max }$ and $L_{\min }, T_{\min }$ are the $\mathrm{TR}$ radiative losses and coronal maximum temperature for solar maximum and minimum respectively (see for example Withbroe 1988). From Fig. 9 it is clear that $\frac{T_{\max }}{T_{\min }} \approx 1.5$ which means that $\frac{L_{\max }}{L_{\min }} \approx 2.8$. It is thus possible that the coronal temperature increase from solar minimum to maximum could have a bearing on the observed solar cycle modulation of TR emission.

\section{Conclusions}

We have observed fundamental elements of the quiet solar corona, namely coronal streamers at solar minimum and solar maximum using the CDS instrument on SOHO. From these observations we have determined the distribution of Fe IX-XV as a function of height out to $2.3 R_{\odot}$ $\left(1.8 R_{\odot}\right)$ at solar minimum (maximum). We find that the solar minimum emission is dominated by Fe XI while at solar maximum the emission is dominated by Fe XV. This comes about due to an order of magnitude increase in the emission measure of Fe XV, while the Fe XI decreases by about half.

We have used the ratio of the most abundant ions to determine the vertical temperature structure at solar minimum and maximum. We find that the temperature increases as a function of height to a peak of about 1.4 MK at $1.3 R_{\odot}$ at solar minimum. This is reduced by around $0.7 \mathrm{MK}$ compared to temperatures determined by both the SOHO CDS and the Yohkoh SXT at solar maximum 22 (see Foley 1998); and the measurements which we present here using CDS for the current maximum 23.

The location of the temperature maximum is coincident with the tops of loops which comprised the regions observed. The temperature declines below and above these loops suggesting they are the focus of energy deposition within coronal streamers. The magnetic field extrapolation suggests that, above the temperature maximum, the field may progressively open with the falling temperature suggesting outflow that may be related to the slow solar wind.

We find that the modulation of coronal electron temperature through the decline and rise of a solar cycle is consistent with a scaling with magnetic field of the order of $B^{1.5}$. This from current theory is consistent with uniform heating by current dissipation and turbulence. We have also shown that this temperature increase could be responsible for the observed solar cycle enhancement of the transition region emission. We plan to investigate these ideas in the future using further observations from the instruments on the SOHO spacecraft. 
Acknowledgements. We are grateful to the UK Particle Physics and Astronomy Research Council for financial support. We acknowledge the Solar UK Research Facility (SURF -http://surfwww.mssl.ucl.ac.uk/surf/) for providing data for use in this publication. SOHO is a project of international cooperation between ESA and NASA. The Yohkoh soft X-ray telescope is a collaborative project of the Lockheed Palo Alto Research Laboratory, the National Astronomical Observatory of Japan, and the University of Tokyo, supported by NASA and ISAS. The Mauna Loa MK3 and MK4 Coronagraph data was provided courtesy, High Altitude Observatory, National Center for Atmospheric Research (NCAR), Boulder, Colorado, USA. NCAR is sponsored by the National Science Foundation.

We would like to thank Terry Kucera, Ron Yurow, Eddie Breeveld, Dave Pike and Stein Vider Haugan for help coordinating and monitoring some of our observations. We would also like to thank Matt Wyndham, Ben Sanderson, Jon Lappington, Alec McCalden, and Alice Breeveld for helpful discussion regarding the way in which the GIS operates, and making this work possible. This research has made use of NASA's Astrophysics Data System Abstract Service. The CHIANTI database is a collaborative project involving the Naval Research Laboratory (Washington DC, USA), the Arcetri Observatory (Firenze, Italy), and Cambridge University (UK). We would also like to thank the anonymous referee for useful comments which improved the clarity of this paper.

\section{References}

Aschwanden, M. J., \& Nitta, N. 2000, ApJ, 535, L59

Breeveld, A. A., Edgar, M. L., Lappington, J. S., \& Smith, A. 1992, SPIE, 1743, 315

Brueckner, G. E., Howard, R. A., Koomen, M. J., et al. 1995, Sol. Phys., 162, 357

Dere, K. P., Landi, E., Mason, H. E., Monsignori Fossi, B. C., \& Young, P. R. 1997, A\&AS, 125, 149

Dupree, A. K., Moore, R. T., \& Shapiro, P. R. 1979, ApJ, 229, L101

Feldman, U., Doschek, G. A., Schühle, U., \& Wilhelm, K. 1999, ApJ, 518, 500

Fisher, R. R., Lee, R. H., MacQueen, M. R., \& Poland. A. I. 1981, Appl. Opt., 20, 1094

Foley, C. R. 1998, Ph.D. Thesis, University of London http://www.mssl.ucl.ac.uk/www_solar/publications/ foleydiss.zip

Foley, C. R., Culhane, J. L., Acton, L. W., \& Lemen, J. 1996, in Proc. of the IAU, 153 (held in Makuhari)
Gabriel, A. H. 1976, Phil. Trans. Roy. Lond. A, 281, 575

Gabryl, J.-R., Cugnon, P., \& Clette, F. 1999, C. Ska, 28, 216

Gibson S. E., Fludra, A., Bagnal, F., et al. JGR, 104, A5, 9691, 1999

Goldstein, B. E., Neugebauer, M., Phillips, J. L., et al. 1996, A\&A, 316, 296

Grevesse, N., \& Sauval, A. J. 1998, Space Sci. Rev., 85, 161

Griffiths, N. W., \& Jordan, C. 1998, ApJ, 497, 883

Guhathakurta, M., Holtzer, T. E., \& MacQueen, R. M. 1996, ApJ, 458, 817

Harrison, R. A., Sawyer, E. C., Carter, M. K., et al. 1995, Sol. Phys., 162, 233

Kopp, R. A., \& Holtzer, T. E. 1976, Sol. Phys., 49, 43

Lang, J., Kent, B. J., Breeveld, A. A., et al. 2000, J. Opt. A: Pure Appl. Opt., vol. 2(2), 88

Landi, E., Del Zanna, G., Breeveld, E. R., et al. 1999, A\&AS, 135,171

Li, J., Raymond, J. C., Acton, L. W., et al. 1998, ApJ, 506, 431

Mazzotta, P., Mazzitelli, G., Colafrancesco, S., \& Vittorio, N. 1998, A\&AS, 133, 403

Mandrini, C. H., Démoulin, P., \& Klimchuk, J. A. 2000, ApJ, 530, 999

Mason, H. E., \& Fossi, B. C. M. 1994, A\&AR, 6, 123

Owocki, S. P., Holzer, T. E., \& Haundhausen, A. J. 1983, ApJ, 275,354

Pevtsov, A. A., \& Acton, L. W. 2001, ApJ, 554, 416

Priest, E. R., Foley, C. R., Heyvaerts, J., et al. 1998, Nature, 393, 545

Priest, E. R., Foley, C. R., Heyvaerts, J., et al. 2000, ApJ, 539, 1002

Raymond, J. C., Kohl, J. L., Noci, G., et al. 1997, Sol. Phys., 175,645

Raymond, J. C., Suleiman, R., \& Kohl, J. L. 1998, Space Sci. Rev., 85, 283

Shull, J. M., \& Van Steenberg, M. 1982, ApJS, 48, 95

Schühle, U., Wilhelm, K., Hollandt, J., Lemaire, P., \& Pauluhn, A. 2000, A\&A, 354, L71

Spitzer, L. 1962, Physics of Fully Ionized Gases (New York: Interscience)

Sturrock, P. A., Wheatland, M. S., \& Acton, L. W. 1996, ApJ, 461, 115

Tsuneta, S., Acton, L., Bruner, M., et al. 1991, Sol. Phys., 136, 37

Wheatland, M. S., Sturrock, P. A., \& Acton, L. W. 1997, ApJ, 482,510

Withbroe, G. L. 1988, ApJ, 325, 442 